\title{
A resistência à insulina no desenvolvimento da doença de Alzheimer: uma revisão narrativa
}

The insulin resistance in the development of Alzheimer's disease: a narrative review

La resistencia a la insulina en el desarrollo de la enfermedad de Alzheimer: una revisión narrativa

Ana Clara da Costa Silva ${ }^{1 *}$, Nathalia Polliana Rodrigues Melgaço ${ }^{1}$, Lilian Caiafa Ferreira Machado ${ }^{1}$, Ana Clara Teixeira Cherem ${ }^{1}$, Bruno Rocha Gelape ${ }^{1}$, Clara Guimarães Carvalho de Oliveira Aquino ${ }^{1}$, José Gabriel Vilhena de Queiroz ${ }^{1}$, Rafaela Charles Correia ${ }^{1}$, Daiane Magalhães ${ }^{1,2,3}$.

\section{RESUMO}

Objetivo: Analisar a influência da resistência à insulina no prognóstico da doença de Alzheimer. Revisão bibliográfica: A doença de Alzheimer é um processo neurodegenerativo, caracterizado pela presença de marcadores patológicos de placas $\beta$-amilóide. A insulina tem função neuromoduladora e no cérebro atua na sobrevivência dos neurônios, na produção de energia, na plasticidade sináptica e na expressão gênica. Estudos recentes revelaram que a resistência à insulina cerebral é um dos fatores de comprometimento cognitivo da doença de Alzheimer. Dessa forma, é de suma importância que se entenda a relação fisiopatológica entre a resistência insulínica e o surgimento da doença de Alzheimer, para que seja possível estabelecer um tratamento mais adequado e evitar o declínio cognitivo através do controle metabólico. Considerações finais: Evidencia-se a existência de uma relação entre o desenvolvimento da doença de Alzheimer e a resistência à insulina e percebe-se a necessidade de melhor entender os mecanismos fisiopatológicos, para que sejam traçadas estratégias terapêuticas adequadas.

Palavras-chave: Doença de Alzheimer, Resistência à insulina, Diabetes mellitus tipo 2.

\begin{abstract}
Objective: Analyze the influence of insulin resistance on the prognosis of Alzheimer's disease. Bibliographic review: Alzheimer's disease is a neurodegenerative process characterized by the presence of pathological markers of $\beta$-amyloid plaques. Insulin has neuromodulatory function and, in the brain, has an important role in the survival of neurons, energy production and gene expression. Recent studies revealed that insulin resistance in the brain is one of the cognitive impairment factors in Alzheimer's disease. Thus, it is critical to understand the pathophysiological relation between insulin resistance and the development of Alzheimer's disease, so that it is possible to establish a more appropriate treatment, and avoid cognitive decline through metabolic control. Final considerations: Stands out the existence of a relationship between the development of Alzheimer's disease and insulin resistance and the need to better understand the pathophysiological mechanisms is perceived, so that adequate therapeutic strategies can be devised.
\end{abstract}

Keywords: Alzheimer disease, Insulin resistance, Type 2 diabetes mellitus.

\footnotetext{
${ }^{1}$ Pontifícia Universidade Católica de Minas Gerais (PUC Minas), Betim - MG.

*E-mail: aanacostta@gmail.com

2 Hospital da Madre Teresa, Belo Horizonte - MG.

${ }^{3}$ Universidade Federal de Minas Gerais (UFMG), Belo Horizonte - MG.
}

SUBMETIDO EM: 11/2021

ACEITO EM: 12/2021

PUBLICADO EM: 1/2022 


\section{RESUMEN}

Objectivo: Analizar la influencia de la resistencia a la insulina en el pronóstico de la enfermedad de Alzheimer. Revisión bibliográfica: La enfermedad de Alzheimer es un proceso neurodegenerativo caracterizado por la presencia de marcadores patológicos de placas $\beta$-amiloides. La insulina tiene una función neuromoduladora y en el cerebro actúa sobre la supervivencia de las neuronas, la producción de energía, la plasticidad sináptica y la expresión genética. Estudios recientes han revelado que la resistencia cerebral a la insulina es uno de los factores de deterioro cognitivo en la enfermedad de Alzheimer. Por lo tanto, es de suma importancia comprender la relación fisiopatológica entre la resistencia a la insulina y la aparición de la enfermedad de Alzheimer para establecer un tratamiento más adecuado y prevenir el deterioro cognitivo mediante el control metabólico. Consideraciones finales: Se evidencia la existencia de una relación entre el desarrollo de la enfermedad de Alzheimer y la resistencia a la insulina, y se percibe la necesidad de conocer mejor los mecanismos fisiopatológicos, para que se puedan delinear estrategias terapéuticas adecuadas.

Palabras clave: Enfermedad de Alzheimer, Resistencia a la insulina, Diabetes mellitus tipo 2.

\section{INTRODUÇÃO}

O termo demência engloba inúmeros sintomas responsáveis por causar um declínio progressivo funcional do paciente. É um quadro abrangente, que engloba perda de memória, de capacidade intelectual, de raciocínio, de competências sociais e alterações das reações emocionais normais. Dentre essas alterações, destaca-se a Doença de Alzheimer (DA), que se caracteriza por uma destruição gradual das áreas cerebrais responsáveis pela capacidade de memória, aprendizagem, raciocínio, julgamento e comunicação. Esse processo neurodegenerativo tem relação com múltiplos fatores e possui uma fisiopatologia complexa. Esses fatores vão desde a atrofia cerebral e danos sinápticos terminais até processos moleculares como a hiperfosforilação da proteína TAU e o depósito exacerbado de $\beta$-amiloides (A $\beta$ ) (BERLANGA-ACOSTA J, et al., 2020).

Observando que a DA é uma das principais causas de morte no mundo e que os mecanismos fisiopatológicos são diversos e ainda necessitam de elucidação, faz-se necessária a exploração das demais influências sistêmicas que afetam o desenrolar dessa patologia (KELLAR D e CRAFT S, 2020). Levando em consideração as informações e os mecanismos supracitados, o Diabetes Mellitus tipo 2 (DM2) se traduz como uma relevante doença, que é caracterizada pela resistência à insulina, hiperglicemia e redução da secreção de insulina pelas células pancreáticas na corrente sanguínea (SOCIEDADE BRASILEIRA DE DIABETES (SBD), 2020). Tal comorbidade está diretamente relacionada a um estilo de vida sedentário e má alimentação, sendo considerada um problema de saúde pública. No Brasil, o diabetes acomete cerca de 12,5 milhões de brasileiros entre a faixa etária de 20 e 79 anos. (INTERNATIONAL DIABETES FEDERATION (IDF) 2017),

Concomitantemente ao predomínio senil gradativo da sociedade, destaca-se a alta prevalência dos distúrbios metabólicos associados, bem como o DM2. Considerando que o cérebro consome cerca de $50 \%$ da glicose para manter seu funcionamento adequado e que esse funcionamento é prejudicado devido a resistência à insulina causada pelo DM2, esta comorbidade apresenta-se como um dos fatores causais de vários quadros demenciais (BERLANGA-ACOSTA J, et al., 2020). O presente trabalho teve como objetivo analisar a influência da resistência à insulina no prognóstico da DA.

\section{REVISÃO BIBLIOGRÁFICA}

\section{Doença de Alzheimer e insulina}

A doença de Alzheimer caracteriza-se pela presença de marcadores patológicos de placas $A \beta$, que podem causar, principalmente, déficits nos neurotransmissores, degeneração dos neurônios, disfunção sináptica, acúmulo extracelular de $A \beta$ e emaranhados neurofibrilares intracelulares (NFT) (NGUYEN TT, et al., 2020). A insulina é um neuromodulador que, no cérebro, influencia na sobrevivência neuronal, na produção de energia, na expressão gênica e na plasticidade sináptica (AKHTAR A e SAH SP, 2020). 
De acordo com a Organização Mundial de Saúde (OMS), estima-se que aproximadamente 50 milhões de pessoas no mundo são diagnosticadas com demência. Especificamente no Brasil, o número de pessoas que vivem com a disfunção passa de um milhão, evidenciando que a doença é uma crise global de saúde. E, apesar de sua patogênese ainda não ser completamente conhecida, a deposição de placa $A \beta$ e a hiperfosforilação da proteína Tau são considerados os principais fatores (KSHIRSAGAR V, et al., 2021). Um diagnóstico de DA, apesar de ser muito difícil de se detectar precocemente, é capaz de modificar tanto a vida de quem a recebe quanto a de seus familiares e amigos. Atualmente, a farmacologia não é capaz de interromper ou retardar a evolução da doença. Com o avanço das pesquisas nas últimas décadas, é possível compreender cada vez mais sobre a forma como que a doença afeta o cérebro, possibilitando dessa maneira buscar possíveis novos manejos não farmacológicos e farmacológicos. (KSHIRSAGAR V, et al., 2021).

Segundo Kshirsagar $V$ et al. (2020), percebe-se que a prevalência de demência é duas vezes maior em pessoas com DM2. De acordo com Gudala K et al. (2013), os diabéticos possuem risco aumentado em $73 \%$ de desenvolver a doença de Alzheimer. O processo metabólico da diabetes exerce influência nos principais domínios da memória, altera a morfologia do cérebro e distorce a comunicação sináptica, que está intimamente relacionada à progressão patológica da DA (AKHTAR A e SAH SP, 2020). Além disso, a DM2 é a comorbidade predominante em pessoas com DA. A resistência à insulina (RI), hiperglicemia e hiperinsulinemia são as principais características em ambas as doenças, que contribuem para a disfunção mitocondrial, o estresse oxidativo e a falha bioenergética (SHARMA VK e SINGH TG, 2020).

Estudos epidemiológicos relacionados à DA revelam que a sinalização desregulada de insulina no cérebro, denominada "resistência à insulina cerebral" é um dos fatores de comprometimento cognitivo e patológico da doença. Este fenômeno pode ser responsável pela progressão da DA. Existem várias moléculas envolvidas nas vias de sinalização da insulina e possíveis irregularidades podem levar à sinalização disfuncional da insulina, contribuindo assim para os sintomas da DA (AKHTAR A e SAH SP, 2020). Alguns estudos epidemiológicos sugerem que a resistência à insulina aumenta o risco de demência e DA, mesmo em populações não diabéticas (NGUYEN TT, et al., 2020). O comprometimento da sinalização da insulina foi observado em pacientes com DA, mesmo naqueles que não eram diabéticos (HÖLSCHER C, 2020).

A insulina é um hormônio produzido e liberado pelas células $\beta$ pancreáticas e, que age tanto no cérebro, quanto nos tecidos periféricos do nosso organismo. Seu transporte para o cérebro é feito por um processo mediado por receptores, através da barreira hematoencefálica $(\mathrm{BHE})$. As células ependimárias e do endotélio cerebral possuem sítios que se ligam à insulina, permitindo, assim, que ela seja transportada. No entanto, mostrou-se que, por o líquido cefalorraquidiano apresentar um nível de insulina não proporcional ao nível encontrado no plasma sanguíneo, esse meio de transporte pode ficar saturado. O cérebro também é uma fonte de insulina e os níveis desse hormônio presente nele podem variar drasticamente, com o hipotálamo e o bulbo olfatório sendo mais concentrados que o plasma sanguíneo (TYAGI A e PUGAZHENTHI S, 2021).

A insulina tem um efeito neuroprotetor e regula a plasticidade sináptica e se torna vital para o funcionamento cognitivo ideal (KSHIRSAGAR V, et al., 2021). O receptor de insulina é expresso principalmente pela quantidade de terminais sinápticos de neurônios no hipocampo, amígdala, hipotálamo, córtex, córtex entorrinal e bulbo olfatório. A DA é caracterizada pela neurodegeneração predominantemente nas regiões cerebrais do hipocampo e epitélio olfatório. Os neurônios têm um papel importante na síntese de insulina devido à presença de RNAm de preproinsulina 1 e 2 nas células neuronais (SHARMA VK e SINGH TG, 2020; ROWLES J et al., 2021).

\section{A resistência à insulina no cérebro}

A resistência à insulina $(\mathrm{RI})$ é o resultado da redução da responsividade dos tecidos periféricos a esse hormônio. Esse acontecimento provoca uma série de mudanças biológicas acompanhadas por um aumento do estresse oxidativo. De forma simplificada, a RI é definida como uma falha nas células cerebrais em responder à entrada de insulina (BERLANGA-ACOSTA J, et al., 2020). Alterações bioenergéticas mediadas por essa resistência podem agravar anormalidades endócrinas na DA, causando acúmulo de placas, redução do hipocampo e distúrbio do metabolismo da glicose cerebrocortical. Além disso, essas mudanças no metabolismo da glicose são prejudiciais na funcionalidade dos neurotransmissores, na neuroplasticidade $e$ em reações inflamatórias (BERLANGA-ACOSTA J, et al., 2020). 
A RI dessensibiliza os mecanismos de insulina através da disfunção do receptor (alteração da expressão e ligação) e da fosforilação anormal (alteração da atividade da quinase). O bloqueio de locais específicos que ocorrem a fosforilação pode ser responsável pela inibição seletiva da ação da insulina, bem como os defeitos na expressão dos receptores de insulina, a fixação do ligante e o movimento da tirosina quinase (SHARMA VK e SINGH TG, 2020; AKHTAR A e SAH SP, 2020).

Evidentemente, a resistência à insulina é exacerbada na DA e na síndrome metabólica devido à inflamação crônica. Foi observado que as concentrações de peptídeo $C$ e insulina estão diretamente correlacionadas e diminuem com o envelhecimento e em indivíduos com DA (TYAGI A e PUGAZHENTHI S, 2021). A neuroinflamação desempenha um papel patológico importante no desenvolvimento da resistência à insulina e à IGF-1 na DA. Assim, a resistência e a deficiência de insulina podem simbolizar um fator crítico que contribui para o desenvolvimento e progressão da DA e da síndrome metabólica (KSHIRSAGAR V, et al., 2020).

Anormalidades nos níveis de glicose surgem devido à resistência dos tecidos à ação da insulina, e à incapacidade do pâncreas de secretar insulina suficiente em níveis normais ou acima do normal para superar a Resistência à Insulina (RI) nos tecidos. A RI aumenta com a idade e o organismo mantém os níveis normais de glicose desde que possa produzir insulina suficiente (AKHTAR A e SAH SP, 2020). A RI leva a diminuição da sinalização da insulina no SNC, seguida por alteração no metabolismo cerebral. Aumento da toxicidade $A \beta$, hiperfosforilação TAU, estresse oxidativo e neuroinflamação são atribuídos à resistência central à insulina, que leva à neurodegeneração (NGUYEN TT, et al., 2020). Embora a hiperinsulinemia e a hiperglicemia possam melhorar a memória agudamente, a longo prazo influenciam no desenvolvimento da DA por meio de combinações de mecanismos como estresse oxidativo, glicosilação de proteínas e isquemia (SHARMA VK e SINGH TG, 2020).

A disfunção metabólica presente na DA e na síndrome metabólica estão associadas a distúrbios neurodegenerativos, sendo a resistência à insulina a principal responsável por essas alterações. Dessa forma, a RI leva à neuroinflamação, disfunção metabólica e mitocondrial, prejuízo na sinalização da insulina na região do cérebro, prejuízo da sinalização da leptina e desregulação do RNAmi. Esses fatores levam à ativação de componentes reativos de oxigênio (ROS) por meio da ativação de citocinas pró-inflamatórias que causam a neurodegeneração (KSHIRSAGAR V, et al., 2021). A citocina induz a presença e o acúmulo do peptídeo $A \beta$ tendo papel importante no desenvolvimento da DA. A deposição de $A \beta$ extracelular, juntamente com Tau hiperfosforilada e NFTs intracelulares são identificados como marcas histopatológicas de DA (BERLANGAACOSTA J, et al., 2020; KSHIRSAGAR V, et al., 2020; AKHTAR A e SAH SP, 2020).

A baixa sinalização de leptina, vai diminuir o potencial de longo prazo e, portanto, a plasticidade sináptica. A leptina, juntamente com a insulina metabólica periférica regulam a expressão do gene miRNA (Ácido ribonucleico mensageiro), e a desregulação desse gene cursa com um aumento de processos apoptóticos e hiperfosforilação da proteína Tau. Dessa maneira sugerindo evidências de que terapias para desordens metabólicas funcionam no nível de miRNA. A transdução do sinal da insulina e da sua interação com os receptores de insulina dependem de um outro componente de membrana chamado gangliosídeos (KSHIRSAGAR V, et al., 2020).

No cérebro humano, o receptor de leptina encontra-se em regiões como o hipotálamo, principalmente no córtex cerebral e no hipocampo, sendo essencial para a cognição e memória. O hormônio também exerce proteção contra a placa beta amiloide e a hiperfosforilação da Tau. No trabalho ainda é citado que a metformina age reduzindo a quantidade de Tau hiperfosforilada e os níveis de JNK fosforilados em ratos obesos resistentes à leptina (KSHIRSAGAR V, et al., 2020).

\section{Receptor de insulina no cérebro}

O receptor de insulina está distribuído por todo o cérebro e em maior densidade no bulbo olfatório, hipotálamo, hipocampo, córtex cerebral e cerebelo. Esse receptor está, em sua maioria, localizado nos neurônios e concentrado nas sinapses em forma de componente da densidade pós-sináptica (PSD). A sinapse é um local importante na sinalização de insulina no cérebro (POMYTKIN I e PINELIS V, 2021). O receptor de insulina é formado por uma transmembrana de tirosina quinase composto por dímeros da subunidade $\alpha / \beta$ que estão ligados por ligações dissulfeto. Esse receptor consiste em uma glicoproteína de 
transmembrana composta por duas subunidades $\alpha$ extracelulares e duas subunidades $\beta$. Esses receptores estão principalmente espalhados pelas regiões do cérebro, tendo concentrações máximas nos núcleos do córtex cerebral e do hipocampo e, em menor quantidade, no bulbo olfatório e hipotálamo (AKHTAR A e SAH SP, 2020; NGUYEN TT, et al., 2020).

Os receptores de insulina nas células endoteliais são essenciais para o transporte da insulina circulante para os tecidos-alvo (TYAGI A e PUGAZHENTHI S, 2021). Em circunstâncias normais, a insulina se liga ao seu receptor, fosforilando-se em resíduos de tirosina que ao final do processo ocorre uma fosforilação dos substratos do receptor de insulina I-IV (IRS) em resíduos de tirosina. A Akt é uma proteína quinase específica para serina-tironina, que tem três subtipos: Akt1, Akt2 e Akt3. A que é expressa nas células do tecido cerebral são as do tipo Akt3, sendo estas regulatórias nos fatores pró-apoptóticos, aumentando a sobrevida celular. (SHARMA VK e SINGH TG, 2020; AKHTAR A e SAH SP, 2020; KELLAR D e CRAFT S, 2020; FERREIRA S, 2021).

A inibição de Akt pode interromper a formação patológica da DA ou controlar o agravamento da doença. Com a redução da ativação da Akt, inicia-se o processo de apoptose neuronal e a insulina no cérebro é capaz de reverter essa apoptose através da ativação da Akt. Portanto, a neurodegeneração neuronal induzida por apoptose presente na doença de Alzheimer pode ser revertida caso a ativação da Akt for aprimorada (AKHTAR A e SAH SP, 2020; BERLANGA-ACOSTA J, et al., 2020).

A DA pode ser caracterizada pela sensibilidade à redução de insulina e a sinalização IRS-1/PI3K desregulada. A PI3K controla eventos metabólicos como a translocação de proteínas transportadoras de glicose, glicogênio e outros componentes (BERLANGA-ACOSTA J, et al., 2020). Assim, a ativação reduzida

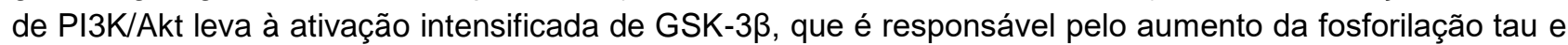
pela deposição aumentada de placas $A \beta$, sendo as duas principais marcas patológicas da DA. $O$ encéfalo de pacientes com DA possuem expressão gênica diminuída de IRS-1 e IRS-2. Além disso, essas expressões gênicas diminuídas se mostraram positivamente associadas à progressão do processo neurodegenerativo (AKHTAR A e SAH SP, 2020; KULAS, 2020).

Em condições de estresse oxidativo, ocorre alterações no funcionamento da PI3K/Akt (complexo de proteína quinase) desempenhando um papel importante na indução da resistência à insulina e prejudicando a plasticidade sináptica. Estudos sobre a manutenção desses mecanismos podem levar a potenciais estratégias terapêuticas para DA e DM2 (SHARMA VK e SINGH TG, 2020).

A sinalização de insulina associada a PI3K está desregulada na DA, portanto, a ativação da PI3K atenua a patologia da DA, aliviando os sintomas da doença. Drogas como acetil-L-carnitina e ácido $\alpha$-lipóico levam à ativação de PI3K, levando à sobrevivência neuronal e o salidroside sinaliza PI3K, tendo ação de neuroproteção. Portanto, sugere-se que a PI3K é um alvo potencial para o tratamento de doenças neurodegenerativas como a DA (AKHTAR A e SAH SP, 2020). Com isso, percebe-se que por meio da estimulação agonística da via PI3K/Akt/GSK-3ß, a insulina evita o acúmulo intraneuronal de $A \beta$ e modula o metabolismo de Tau (BERLANGA-ACOSTA J, et al., 2020; ZHENG M e WANG P, 2021).

A insulina também atua no acúmulo de receptores GABA-A nas membranas pós-sinápticas e participa da tradução da proteína pós-sináptica PSD-95. A RI afeta a transmissão de GABA ao recrutar receptores GABA funcionais para o local pós-sináptico (HÖLSCHER C, 2020). Esse acúmulo leva ao desenvolvimento da resistência à insulina e à regulação negativa dos receptores de insulina em cérebros com DA. Como a doença de Alzheimer forma uma resistência específica à insulina, já é considerada por muitos estudos "diabetes tipo 3" (KSHIRSAGAR V et al., 2020; MEJIDO, 2020).

Alguns gangliosídeos, como o GM3, são reguladores da resistência insulínica. Já o GM1 que também possui uma relação com a toxicidade dos agregados de $A \beta$, sendo que quando ocorre uma mudança na conformação lipídica o GM1 se acumula e gera um aumento de oligômeros de beta-amiloides (AßOs) por meio da formação do complexo $\mathrm{GAB}$, que é a associação de um peptídeo $\mathrm{AB}$ com um gangliosídeos. Sabese, portanto, que a insulina reduz os níveis de GM1 e consequentemente a deposição de placas. Tal conhecimento e associação pode levar a estratégia terapêutica potencial de manejo de DA e DM2 associados à resistência insulínica (KSHIRSAGAR V, et al., 2020). 


\section{Abordagens terapêuticas}

A insulina desempenha um papel crucial na saúde do cérebro e a desregulação da insulina pode contribuir para as condições de envelhecimento cerebral patológico (KELLAR D e CRAFT S, 2020). Embora os estudos com insulina tenham mostrado bons resultados, essa não é uma droga ideal para pacientes que possuem DA. O prognóstico a longo prazo pode causar níveis elevados desse hormônio e aumentar sua resistência e sensibilização, agravando o caso de DA. Os hormônios incretina (GLP-1 e GIP) são análogos da insulina e não aumentam a dessensibilização à esta, pois não ativam seus receptores, em vez disso, podem dessensibilizar a sinalização de insulina. Por tanto, esse tratamento é indicado em pacientes com DA não diabéticos. Os agonistas do receptor GLP-1 do hormônio incretina são tratamentos medicamentosos eficazes e bem tolerados para o DM2 (HÖLSCHER C, 2020; ALVES S, et al., 2021).

Um tratamento que está obtendo resultados promissores é a administração de insulina no SNC através da via intranasal (IN). Alguns estudos indicam que a administração de insulina IN foi responsável pela melhora na memória tardia, que tem ação do hipocampo. A melhora na memória foi intensificada pela administração de insulina aspart, análoga à insulina. A administração de insulina no SNC por via IN tem um fator importante no controle da homeostase periférica da glicose, melhorando assim a função cognitiva em pacientes amnésicos. As deficiências na sensibilidade sistêmica e cerebral à insulina estão inter relacionadas e podem contribuir para a patogênese e progressão da DA (HALLSCHMID M, 2021; DUBEY SK, 2020; BURILO J, 2021; ARVANITAKIS Z, 2020).

\section{CONSIDERAÇÕES FINAIS}

Diante dessas informações, conclui-se que, apesar de necessitar de elucidações a respeito da fisiopatologia, a relação entre o descontrole metabólico em pacientes diabéticos e o surgimento da DA existe. Como a insulina possui efeito neuroprotetor, o descontrole da regulação desse hormônio é um fator que pode contribuir para o envelhecimento cerebral de forma patológica e o agravamento de quadros demenciais. Sendo assim, o conhecimento dos mecanismos envolvidos nesse processo pode favorecer o desenvolvimento de estratégias terapêuticas que visem a interrupção da progressão e a melhora da qualidade de vida de pacientes com a doença de Alzheimer.

\section{AGRADECIMENTOS E FINANCIAMENTO}

A Liga Acadêmica de Neurociências pela mobilização e participação conjunta que viabilizou este estudo. Ao Diretório Acadêmico Horizontal (DAHMP) da PUC Minas Betim por desenvolver o programa de financiamento de artigos e apoiar a publicação do estudo.

\section{REFERÊNCIAS}

1. ALVES SS, et al. Insulin Resistance as a Common Link Between Current Alzheimer's Disease Hypotheses. Journal of Alzheimer's Disease, 2021; 1-35.

2. AKHTAR A, SAH SP. Insulin signaling pathway and related molecules: role in neurodegeneration and Alzheimer's disease. Neurochemistry international, 2020; 135; 104707.

3. ARVANITAKIS Z, et al. Brain Insulin Signaling, Alzheimer Disease Pathology, and Cognitive Function. Annals of Neurology, 2020; 88: 513-525.

4. BERLANGA-ACOSTA J, et al. Insulin Resistance at the Crossroad of Alzheimer Disease Pathology: A Review. Frontiers in Endocrinology, 2020; 11; 560375.

5. BURILLO J, et al. Insulin Resistance and Diabetes Mellitus in Alzheimer's Disease. Cells, 2021; $10(5): 1236$.

6. DUBEY SK, et al. Insulin mediated novel therapies for the treatment of Alzheimer's disease. Life Sciences, 2020; 249; 117540.

7. FERREIRA ST. Brain insulin, insulin-like growth factor 1 and glucagon-like peptide 1 signalling in Alzheimer's disease. Journal of Neuroendocrinology, 2021; 33(4); e12959.

8. HALLSCHMID M. Intranasal insulin for Alzheimer's disease. CNS drugs, 2021; 35(1):21-37.

9. HÖLSCHER C. Brain insulin resistance: role in neurodegenerative disease and potential for targeting. Expert opinion on investigational drugs, 2020; 29(4); 333-348. 
10. INTERNATIONAL DIABETES FEDERATION. IDF Clinical Practice Recommendations for managing Type 2 Diabetes in Primary Care. IDF, 2017. Disponível em: < https://www.idf.org/e-library/guidelines/128-idf-clinical-practicerecommendations-for-managing-type-2-diabetes-in-primary-care.html >. Acessado em: 23 de junho de 2021.

11. KELLAR D, CRAFT S. Brain insulin resistance in Alzheimer's disease and related disorders: mechanisms and therapeutic approaches. The Lancet Neurology, 2020; 19(9); 758-766.

12. KULAS JA, et al. Insulin Resistance and Impaired Lipid Metabolism as a Potential Link Between Diabetes and Alzheimer's Disease. HHS Author Manuscripts, 2020; 81(2): 194-205.

13. KSHIRSAGAR V, et al. Insulin resistance: a connecting link between Alzheimer 's disease and metabolic disorder. Metabolic Brain Disease, 2020; 36(1); 67-83.

14. MEJIDO DCP, et al. Insulin and leptin as potential cognitive enhancers in metabolic disorders and Alzheimer's disease. Neuropharmacology, 2020; 171; 108115.

15. NGUYEN TT, et al. Role of Insulin Resistance in the Alzheimer's Disease Progression. Neurochemical research, 2020; 45(7); $1481-1491$.

16. POMYTKIN I, PINELIS V. Brain Insulin Resistance: Focus on Insulin Receptor-Mitochondria Interactions. Life, 2021; $11(3) ; 262$.

17. ROWLES JE, et al. Are heat shock proteins an important link between type 2 diabetes and Alzheimer disease? International Journal of Molecular Sciences, 2020; 2(21); 8204.

18. SHARMA VK, SINGH TG. Insulin resistance and bioenergetic manifestations: Targets and approaches in Alzheimer's disease. Life Sciences, 2020; 262; 118401.

19. SOCIEDADE BRASILEIRA DE DIABETES. Diretrizes da Sociedade Brasileira de Diabetes (2019-2020). São Paulo, 2020. Disponível em: < http://www.saude.ba.gov.br/wp-content/uploads/2020/02/Diretrizes-Sociedade-Brasileira-deDiabetes-2019-2020.pdf >. Acessado em: 23 de junho de 2021.

20. TYAGI A, PUGAZHENTHI S. Targeting Insulin Resistance to Treat Cognitive Dysfunction. Molecular Neurobiology, $2021 ; 58(6) ; 1-20$.

21. ZHENG M, WANG P. Role of insulin receptor substance-1 modulating PI3K/Akt insulin signaling pathway in Alzheimer's disease. 3 Biotech, 2021; 11(4); 1-17. 